
\title{
28 Research Square \\ Using Plasmonic Cloaking Method on Infinite Cylindrical Structures and its Applications
}

\section{Afsaneh Rezaei}

Shiraz University

Farzad Mohajeri ( $\triangle$ mohajeri@shirazu.ac.ir)

Shiraz University https://orcid.org/0000-0002-0444-7614

\section{Zahra Hamzavi Zarghani}

Shiraz University

\section{Research Article}

Keywords: Plasmonic, Metamaterial, Plasmonic cloak, Reverse polarization

Posted Date: June 9th, 2021

DOl: https://doi.org/10.21203/rs.3.rs-494808/v1

License: (1) This work is licensed under a Creative Commons Attribution 4.0 International License. Read Full License

Version of Record: A version of this preprint was published at Journal of Computational Electronics on October 9th, 2021. See the published version at https://doi.org/10.1007/s10825-021-01787-4. 


\title{
Using Plasmonic Cloaking Method on Infinite Cylindrical
}

\section{Structures and its Applications}

\author{
Afsaneh Rezaei ${ }^{1}$, Farzad Mohajeri, ${ }^{2, *}$,Zahra Hamzavi-Zarghani ${ }^{3}$ \\ ${ }^{1}$ M.Sc., Department of Communications and Electronics, School of Electrical and Computer Engineering, Shiraz \\ University, Shiraz, Iran. \\ ${ }^{2}$ Associate Professor, Department of Communications and Electronics, School of Electrical and Computer \\ Engineering, Shiraz University, Shiraz, Iran. \\ ${ }^{3}$ Ph.D., Department of Communications and Electronics, School of Electrical and Computer Engineering, Shiraz \\ University, Shiraz, Iran. \\ *Corresponding author. \\ E-mail: mohammad.fendereski@gmail.com (A.Rezaei), mohajeri@shirazu.ac.ir (F.Mohajeri), \\ zahrahamzavi@yahoo.com (Z.Hamzavi-Zarghani).
}

\begin{abstract}
In recent years, cloaking using materials with negative electric permittivity or magnetic permeability has been studied and researched. It has been demonstrated that covering an object with a cloak that has negative electric permittivity or magnetic permeability or less than one can cause a reduction of the scattering cross section (SCS) of the object. In this paper, we have solved the problem of scattering for the object and single-layer or multi-layer cylindrical cloaks and by doing so, we have obtained the necessary fundamental equations for designing these types of cloaks in two conditions, with and without considering the effects of coupling on the mathematics of solving the problem of scattering. Using the obtained equations we have demonstrated that by using this technique reducing the level of visibility of the object is possible.
\end{abstract}

Keywords: Plasmonic; Metamaterial; Plasmonic cloak; Reverse polarization.

\section{Introduction}

Human interest in becoming invisible can be found from many years ago in stories of different nations. This human ambition has not been abandoned during the long centuries. Nowadays, cloaking is not an unreachable dream anymore as a result of the advancement of science. Researchers have been looked for an answer to this question that can a cloaking layer be achieved to make an object invisible at least in the limited range of frequency? In 1961, Dolin investigated anisotropic nonhomogeneous structures and showed that by using these kinds of structures the electromagnetic wave will pass through the object without perturbation [1]. Other structures have been introduced by Pendry (2006) [2] and Greenleaf (2009) [3]. Also, Kerker has been published a paper titled "Cloaked Objects" in 1975 which inspires Engheta and Alú for invisibility based on 
the scattering cancellation method [4]. With the advent of the metamaterials and advancement of technology in the fabrication of artificial materials with extraordinary electromagnetic properties, a new arena for realization of cloaking emerged and a lot of researchers have been attracted to the use of metamaterials for invisibility purposes.

An electromagnetic cloaking layer is a device that is capable to make the objects exposed to the electromagnetic waves invisible in a determined range of frequency. Cloaking in physics means that the cloaked object does not produce any changes in the fields around it. In other words, the cloaked object does not produce reflected waves to the source or scattered waves in other directions. Moreover, it does not absorb the waves. But invisibility in electromagnetic waves scattering theory is the same as achieving near zero or ideally exactly zero SCS. Since SCS is defined as the ratio of the scattered power density of the object to the incident power density, then zero SCS means invisibility.

Several methods to achieve invisibility have been introduced until now, each of them has some advantages and disadvantages. Some of the most famous methods are the Coordinate Transformation Technique [5], Transmission Line Techniques [6,7], Parallel Plates Cloaking [8], Hybrid Method [9] and Active Schemes [10]. Since in the majority of these methods anisotropic and inhomogeneous materials are used, the realization of the cloaking layer was impossible in practice. However, after introducing plasmonic cloaking and fabrication feasibility of the cloaking layer, a new era has emerged. In 2005, Engheta and Alú, have presented a new technique in a paper titled "Achieving Transparency with Plasmonic and Metamaterial Coatings" which is realizable in practice because of using isotropic and homogeneous materials [11]. In this method which is based on the scattering cancellation method and is called as "Plasmonic Cloaking" invisibility is obtained utilizing materials with the electric permittivity or magnetic permeability less than one or negative. For instance, some metals near their plasma frequencies or metamaterials with negative parameters can be used [12]. It is worth noting that the idea of using the layers with near zero electric permittivity for invisibility purpose in quasi-static conditions for very small spheres ( having the radius much smaller than the wavelength) has been introduced before [13]. However, in the method known as plasmonic cloaking, the scattering problem can be solved for more general cases (dynamic and non-static).

The main idea of achieving invisibility with a plasmonic cloak is a significant reduction of SCS of the collection of the object and the cloaking layer. We know that the bigger size of the object, the larger SCS. Therefore, it is expected the SCS increases by adding the plasmonic layer to the object. However, in the plasmonic cloaking method, the SCS reduces by adding a properly designed layer with negative or near zero electric permittivity or magnetic permeability. Therefore, with optimized designing of the cloaking layer parameters, SCS of the collection can be reached to zero which results in invisibility.

Here, a physical justification of the plasmonic cloaking operation is presented. According to Fig. 1 a spherical object with dielectric constant $\varepsilon_{1}>\varepsilon_{0}$ is assumed which is covered by a layer with dielectric constant $\varepsilon_{2}<\varepsilon_{0}$ which is illuminated by an electromagnetic wave. 


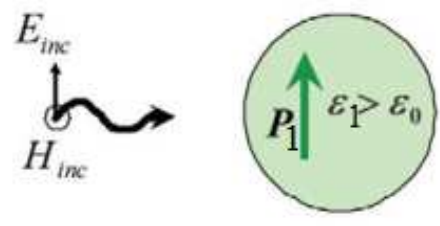

(a) $\quad \mathbf{P}_{1}=\left(\varepsilon_{1}-\varepsilon_{0}\right) \mathbf{E}_{\text {inc }}$

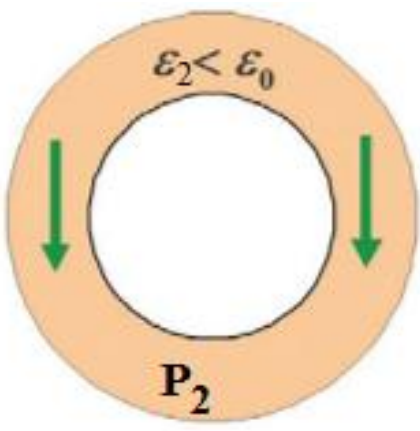

(b) $\quad \mathbf{P}_{2}=\left(\varepsilon_{2}-\varepsilon_{0}\right) \mathbf{E}$ inc

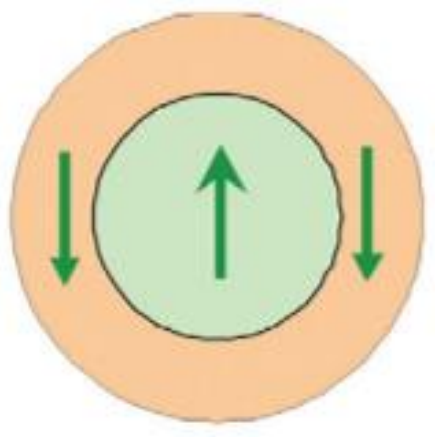

(c) $\mathrm{SCS} \approx 0$

Fig.1. (a) spherical object with dielectric constant more than 1, (b) spherical object covered by a layer with dielectric constant less

than 1, (c) cancellation of the object and layer's polarizations vector and significant reduction of SCS.

Plasmonic cloaking has several advantages compared to previous methods such as: non-resonant nature, more bandwidth [14], robustness of layer which means a small change in the geometry or frequency will not ruin the cloaking operation [15], obtaining transparency in both near and far field for one or some particles [16], the penetration of the field into the object and not being isolated from the environment which allows the cloaked object to be used in measurement sensors [17].

In 2013, Farhat and his colleagues presented the possibility of achieving a controllable cloaking layer using graphene in a paper. They show that by biasing the grapheme layers with an external dc voltage, it is possible to obtain a tuneable cloaking layer at different frequencies [18]. Another advantage of graphene in plasmonic cloaking technique is its easy realization in practice.

So far, most research has focused on invisibility for spherical objects and less has been done on cylindrical structures [19]. This paper tries to address the issue of invisibility in an infinite cylindrical structures.

\section{Infinite cylindrical structures with multilayer cloak}

Engheta and Alú have introduced a two-layer plasmonic cloak to achieve invisibility at some different frequencies [14]. Our goal is to investigate the scattering problem of a multilayer plasmonic cloak in cylindrical structures with plane wave excitation. At first, we consider the desired number of the layers and obtain the scattering coefficients in a general case. It is worth noting that by utilizing multilayer cloaks, not only obtaining multi-frequency cloak can be achieved but also reducing the scattering coefficients for the higher harmonics is possible. Because adding each layer to the structure leads to increasing the degrees of freedom in design procedure which results in the possibility of minimizing the higher harmonics simultaneously. Fig. 2 shows a cross section of a multilayer cloak to make the cylindrical structure, invisible. 

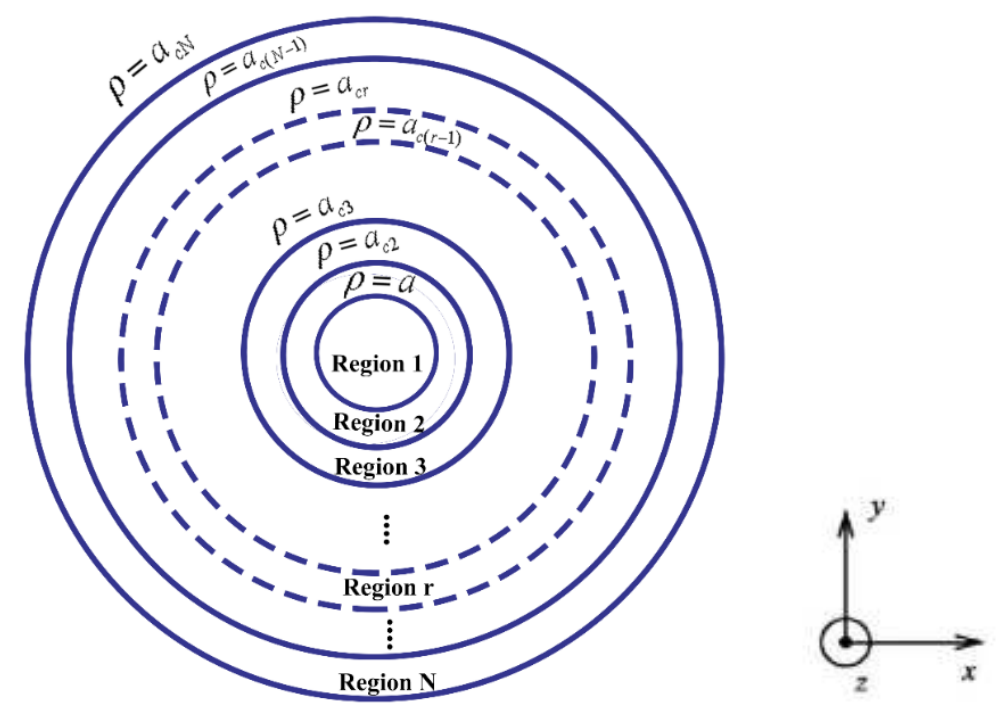

Fig. 2. The cross section of a multilayer cloak for an infinite cylinder.

The number of cloaking layers is N-1 and their axis is in the $z$ axis. The radius of the considered invisible cylinder is "a" and its electric permittivity and magnetic permeability are " $\varepsilon$ " and " $\mu$ ", respectively. The radius of the $r^{\text {th }}$ cylinder is "acr" and its electric permittivity and magnetic permeability are " $\varepsilon$ cr" and " $\mu_{\mathrm{cr}}$ ", respectively.

This problem can be solved for TE or TM polarization. Here we continue our investigation on scattering of cylinders for TM polarization is much higher than TE polarization.

It is supposed that a $\mathrm{TM}_{\mathrm{z}}$ polarized plane wave with time dependence of $e^{-j \omega t}$ incidents to the structure of Fig. 2. The incident wave vector can be written in the form of eq. (1) [20].

$$
\boldsymbol{E}^{\boldsymbol{i}}=E_{0} \hat{a}_{z} e^{-j k_{0} x}
$$

Where $k_{0}=\omega \sqrt{\varepsilon_{0} \mu_{0}}$ is the wavenumber in the air.

By transforming the Cartesian coordinates to the cylindrical one, the tangential incident electric field can be written in the form of eq. (2) [20].

$$
E_{z}^{i}=\mathrm{E}_{0} \sum_{-\infty}^{+\infty} j^{-n} J_{n}\left(k_{0} \rho\right) e^{j n \varphi}
$$

The total tangential electric field in the air consists of two parts: the electric field of the incident wave and scattered wave. The electric field of the scattered wave in the $\mathrm{z}$ direction can be written according to eq. (3).

$$
E_{Z}^{\text {scat }}=\mathrm{E}_{0} \sum_{-\infty}^{+\infty} C_{s, n}^{\mathrm{TM}} j^{-n} H_{n}^{(2)}\left(k_{0} \rho\right) e^{j n \varphi}
$$

Where $H_{n}^{(2)}$ is the Hankel function of the second kind. For the special case of normal incidence, the electric field in the all layers are written as follows:

$$
\begin{aligned}
& E_{z}^{1}=\mathrm{E}_{0} \sum_{-\infty}^{+\infty} j^{-n} C_{1, n}^{\mathrm{TM}} J_{n}(k \rho) e^{j n \varphi} \\
& E_{z}^{2}=\mathrm{E}_{0} \sum_{-\infty}^{+\infty} j^{-n}\left[C_{2 i, n}^{\mathrm{TM}} J_{n}\left(k_{c 2} \rho\right)+C_{2 o, n}^{\mathrm{TM}} Y_{n}\left(k_{c 2} \rho\right)\right] e^{j n \varphi} \\
& E_{z}^{3}=\mathrm{E}_{0} \sum_{-\infty}^{+\infty} j^{-n}\left[C_{3 i, n}^{\mathrm{TM}} J_{n}\left(k_{c 3} \rho\right)+C_{3 o, n}^{\mathrm{TM}} Y_{n}\left(k_{c 3} \rho\right)\right] e^{j n \varphi}
\end{aligned}
$$




$$
\begin{aligned}
& E_{z}^{r}=\mathrm{E}_{0} \sum_{-\infty}^{+\infty} j^{-n}\left[C_{r i, n}^{\mathrm{TM}} J_{n}\left(k_{c r} \rho\right)+C_{r o, n}^{\mathrm{TM}} Y_{n}\left(k_{c r} \rho\right)\right] e^{j n \varphi} \\
& E_{z}^{N}=\mathrm{E}_{0} \sum_{-\infty}^{+\infty} j^{-n}\left[C_{N i, n}^{\mathrm{TM}} J_{n}\left(k_{c N} \rho\right)+C_{N o, n}^{\mathrm{TM}} Y_{n}\left(k_{c N} \rho\right)\right] e^{j n \varphi}
\end{aligned}
$$

Where $\mathrm{J}_{\mathrm{n}}$ and $\mathrm{Y}_{\mathrm{n}}$ are Bessel functions of the first kind and second kind, respectively, $C_{s, n}^{T M}$ are scattering coefficients, $k=$ $\omega \sqrt{\mu \varepsilon}$ and $k_{c r}=\omega \sqrt{\mu_{c r} \varepsilon_{c r}}$ are the wavenumbers in cylindrical layers.

It is worth noting that because we neglect the mutual coupling, the magnetic field of the scattered wave in the $\mathrm{z}$ direction is zero $\left(H_{Z}^{\text {scat }}=0\right)$.

To obtain unknown coefficients in eq. (4), continuity of tangential electric and magnetic fields should be applied in all intersections. The tangential magnetic fields are calculated from the Maxwell's equations as follows [20]:

$$
H_{\varphi}=-\frac{1}{j \omega \mu}\left[\frac{\partial}{\partial z}\left(-\frac{1}{j \omega \varepsilon} \frac{\partial H \varphi}{\partial z}\right)-\frac{\partial E_{z}}{\partial \rho}\right]
$$

By applying the boundary conditions at the intersections $\rho=\mathrm{a}, \mathrm{ac2}, \mathrm{ac} 3 \ldots \mathrm{acN}$, following $2 \mathrm{~N}$ equations are obtained:

$$
\begin{aligned}
& C_{2 i, n}^{\mathrm{TM}} J_{n}\left(k_{c 2} a\right)+C_{2 o, n}^{\mathrm{TM}} Y_{n}\left(k_{c 2} a\right)=C_{1, n}^{\mathrm{TM}} J_{n}(k a) \\
& C_{2 i, n}^{\mathrm{TM}} J_{n}\left(k_{c 2} a_{c 2}\right)+C_{2 o, n}^{\mathrm{TM}} Y_{n}\left(k_{c 2} a_{c 2}\right)=C_{3 i, n}^{\mathrm{TM}} J_{n}\left(k_{c 3} a_{c 2}\right)+C_{3 o, n}^{\mathrm{TM}} Y_{n}\left(k_{c 3} a_{c 2}\right) \\
& C_{3 i, n}^{\mathrm{TM}} J_{n}\left(k_{c 3} a_{c 3}\right)+C_{3 o, n}^{\mathrm{TM}} Y_{n}\left(k_{c 3} a_{c 3}\right)=C_{4 i, n}^{\mathrm{TM}} J_{n}\left(k_{c 4} a_{c 3}\right)+C_{4 o, n}^{\mathrm{TM}} Y_{n}\left(k_{c 4} a_{c 3}\right) \\
& C_{r i, n}^{\mathrm{TM}} J_{n}\left(k_{c r} a_{c r}\right)+C_{r o, n}^{\mathrm{TM}} Y_{n}\left(k_{c r} a_{c r}\right)=C_{(r+1) i, n}^{\mathrm{TM}} J_{n}\left(k_{c(r+1)} a_{c r}\right)+C_{(r+1) o, n}^{\mathrm{TM}} Y_{n}\left(k_{c(r+1)} a_{c r}\right) \\
& C_{N i, n}^{\mathrm{TM}} J_{n}\left(k_{c N} a_{c N}\right)+C_{N o, n}^{\mathrm{TM}} Y_{n}\left(k_{c N} a_{c N}\right)=J_{n}\left(k_{0} a_{c N}\right)+C_{s, n}^{\mathrm{TM}} H_{n}^{(2)}\left(k_{0} a_{c N}\right) \\
& \frac{1}{\eta_{c 2}} C_{2 i, n}^{\mathrm{TM}} J^{\prime}{ }_{n}\left(k_{c 2} a\right)+\frac{1}{\eta_{c 2}} C_{2 o, n}^{\mathrm{TM}} Y^{\prime}{ }_{n}\left(k_{c 2} a\right)=\frac{1}{\eta} C_{1, n}^{\mathrm{TM}} J_{n}^{\prime}(k a) \\
& \frac{1}{\eta_{c 2}} C_{2 i, n}^{\mathrm{TM}} J_{n}^{\prime}\left(k_{c 2} a_{c 2}\right)+\frac{1}{\eta_{c 2}} C_{2 o, n}^{\mathrm{TM}} Y^{\prime}{ }_{n}\left(k_{c 2} a_{c 2}\right)=\frac{1}{\eta_{c 3}} C_{3 i, n}^{\mathrm{TM}} J^{\prime}{ }_{n}\left(k_{c 3} a_{c 2}\right)+\frac{1}{\eta_{c 3}} C_{3 o, n}^{\mathrm{TM}} Y^{\prime}{ }_{n}\left(k_{c 3} a_{c 2}\right) \\
& \frac{1}{\eta_{c 3}} C_{3 i, n}^{\mathrm{TM}} J^{\prime}{ }_{n}\left(k_{c 3} a_{c 3}\right)+\frac{1}{\eta_{c 3}} C_{3 o, n}^{\mathrm{TM}} Y^{\prime}{ }_{n}\left(k_{c 3} a_{c 3}\right)=\frac{1}{\eta_{c 4}} C_{4 i, n}^{\mathrm{TM}} J^{\prime}{ }_{n}\left(k_{c 4} a_{c 3}\right)+\frac{1}{\eta_{c 4}} C_{4 o, n}^{\mathrm{TM}} Y^{\prime}{ }_{n}\left(k_{c 4} a_{c 3}\right) \\
& \frac{1}{\eta_{c r}} C_{r i, n}^{\mathrm{TM}} J^{\prime}{ }_{n}\left(k_{c r} a_{c r}\right)+\frac{1}{\eta_{c r}} C_{r o, n}^{\mathrm{TM}} Y_{n}^{\prime}{ }_{n}\left(k_{c r} a_{c r}\right)=\frac{1}{\eta_{c(r+1)}} C_{(r+1) i, n}^{\mathrm{TM}} J^{\prime}{ }_{n}\left(k_{c(r+1)} a_{c r}\right)+\frac{1}{\eta_{c(r+1)}} C_{(r+1) o, n}^{\mathrm{TM}} Y_{n}^{\prime}\left(k_{c(r+1)} a_{c r}\right) \\
& \frac{1}{\eta_{c N}} C_{N i, n}^{\mathrm{TM}} J^{\prime}{ }_{n}\left(k_{c N} a_{c N}\right)+\frac{1}{\eta_{c N}} C_{N o, n}^{\mathrm{TM}} Y^{\prime}{ }_{n}\left(k_{c N} a_{c N}\right)=\frac{1}{\eta_{0}} J^{\prime}{ }_{n}\left(k_{0} a_{c N}\right)+\frac{1}{\eta_{0}} C_{s, n}^{\mathrm{TM}}{H^{\prime}}_{n}^{(2)}\left(k_{0} a_{c N}\right)
\end{aligned}
$$

Where $J_{n}^{\prime}$ and $Y_{n}{ }^{\prime}$ are derivative of Bessel functions of the first and second kinds respectively, $H_{n}^{\prime(2)}$ is derivative of Hankel function of the second kind with respect to the argument and $\eta=\sqrt{\frac{\mu}{\varepsilon}}$ and $\eta_{c r}=\sqrt{\frac{\mu_{c r}}{\varepsilon_{c r}}}$ are intrinsic impedances in the layers. 
Equations (6) consists of the system of linear and inhomogeneous equations with $2 \mathrm{~N}$ unknown coefficients. By solving these equations, the scattering coefficients are obtained as:

$$
C_{s, n}^{\mathrm{TM}}=\frac{\left|\Delta^{\prime}\right|}{|\Delta|}
$$

\section{Where}

$$
\Delta=\left[\begin{array}{l}
A_{1} \\
A_{2} \\
A_{3} \\
\vdots \\
A_{r} \\
\vdots \\
A_{N-1} \\
A_{N}
\end{array}\right]
$$

\section{And}

$$
\Delta^{\prime}=\left[\begin{array}{l}
A_{1} \\
A_{2} \\
A_{3} \\
\vdots \\
A_{r} \\
\vdots \\
A_{N-1} \\
B_{N}
\end{array}\right]
$$

Where the coefficients $A_{1}, A_{2}, \ldots, A_{r}, \ldots, A_{N}$ and $B_{N}$ are $2 \times 2 N$ matrices that evaluated as follows:

$$
\begin{aligned}
& A_{1}=\left[\begin{array}{cclllll}
-J_{n}(k a) & J_{n}\left(k_{c 2} a\right) & Y_{n}\left(k_{c 2} a\right) & 0 & 0 & \ldots & 0 \\
-\frac{1}{\eta} J^{\prime}{ }_{n}(k a) & \frac{1}{\eta_{c 2}} J^{\prime}{ }_{n}\left(k_{c 2} a\right) & \frac{1}{\eta_{c 2}} Y^{\prime}{ }_{n}\left(k_{c 2} a\right) & 0 & 0 & \ldots & 0
\end{array}\right] \\
& A_{2}=\left[\begin{array}{llllllll}
0 & J_{n}\left(k_{c 2} a_{c 2}\right) & Y_{n}\left(k_{c 2} a_{c 2}\right) & -J_{n}\left(k_{c 3} a_{c 2}\right) & -Y_{n}\left(k_{c 3} a_{c 2}\right) & 0 & \ldots & 0 \\
0 & \frac{1}{\eta_{c 2}} J^{\prime}{ }_{n}\left(k_{c 2} a_{c 2}\right) & \frac{1}{\eta_{c 2}} Y^{\prime}{ }_{n}\left(k_{c 2} a_{c 2}\right) & -\frac{1}{\eta_{c 3}} J^{\prime}{ }_{n}\left(k_{c 3} a_{c 2}\right) & -\frac{1}{\eta_{c 3}} Y^{\prime}{ }_{n}\left(k_{c 3} a_{c 2}\right) & 0 & \ldots & 0
\end{array}\right]
\end{aligned}
$$

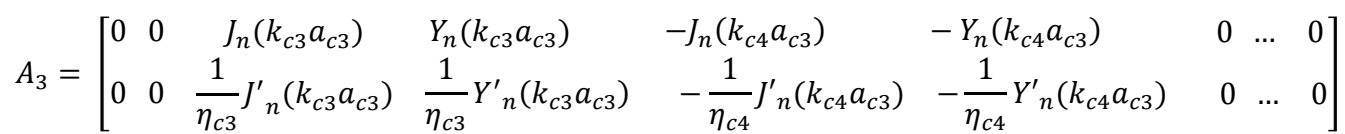

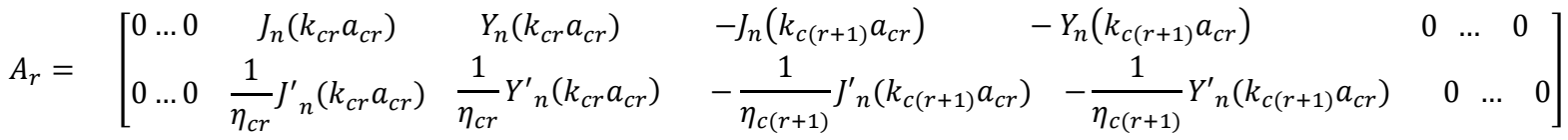




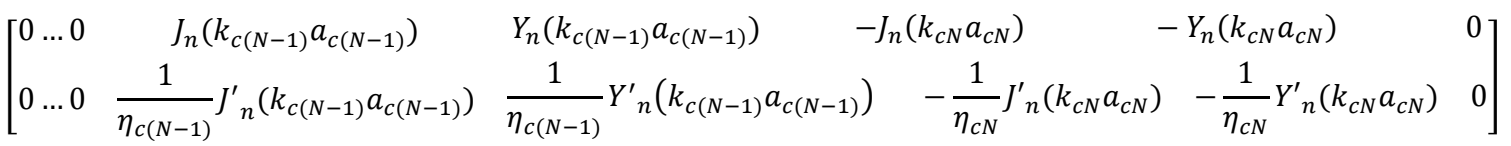

$$
\begin{aligned}
& A_{N}=\left[\begin{array}{ccccccc}
0 & 0 & \ldots & 0 & J_{n}\left(k_{c N} a_{c N}\right) & Y_{n}\left(k_{c N} a_{c N}\right) & -H_{n}^{(2)}\left(k_{0} a_{c N}\right) \\
0 & 0 & \ldots & 0 & \frac{1}{\eta_{c N}} J^{\prime}{ }_{n}\left(k_{c N} a_{c N}\right) & \frac{1}{\eta_{c N}} Y^{\prime}{ }_{n}\left(k_{c N} a_{c N}\right) & -\frac{1}{\eta_{0}} H^{\prime}{ }_{n}^{(2)}\left(k_{0} a_{c N}\right)
\end{array}\right] \\
& B_{N}=\left[\begin{array}{rrrrrrr}
0 & 0 & \ldots & 0 & J_{n}\left(k_{c N} a_{c N}\right) & Y_{n}\left(k_{c N} a_{c N}\right) & J_{n}\left(k_{0} a_{c N}\right) \\
0 & 0 & \ldots & 0 & \frac{1}{\eta_{c N}} J^{\prime}{ }_{n}\left(k_{c N} a_{c N}\right) & \frac{1}{\eta_{c N}} Y^{\prime}{ }_{n}\left(k_{c N} a_{c N}\right) & \frac{1}{\eta_{0}} J^{\prime}{ }_{n}\left(k_{0} a_{c N}\right)
\end{array}\right]
\end{aligned}
$$

\section{Simulation results}

In this section, simulation results for a dielectric cylinder covered by two cloaking layers which is shown in Fig. 3 are investigated.

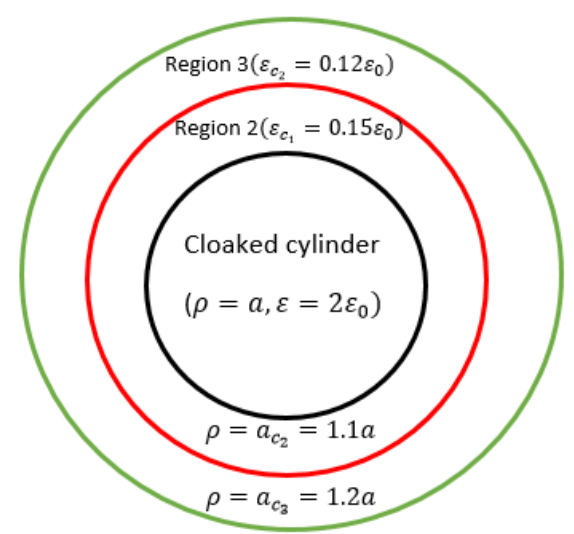

Fig. 3. Dielectric cylinder covered by two cloaking layers.

Figs. 4 and 5 show normalized scattering cross section (SCS) for the cloaked cylinder with two values of radius, a=10 nm and $\mathrm{a}=30 \mathrm{~nm}$, respectively and dielectric constant 2 . Also $\mathrm{a}_{\mathrm{c} 2}=1.1 \mathrm{a}$ and $\mathrm{a}_{\mathrm{c} 3}=1.2 \mathrm{a}$ are the radii of the first and second covering layers. Values of electric permittivity coefficients of this layers, by using Drude model and in desired frequencies are $\varepsilon_{c_{1}}=$ $0.15 \varepsilon_{0}$ and $\varepsilon_{c_{2}}=0.12 \varepsilon_{0}$ respectively. Also the losses of two layers is in the acceptable range in the Drude model. 


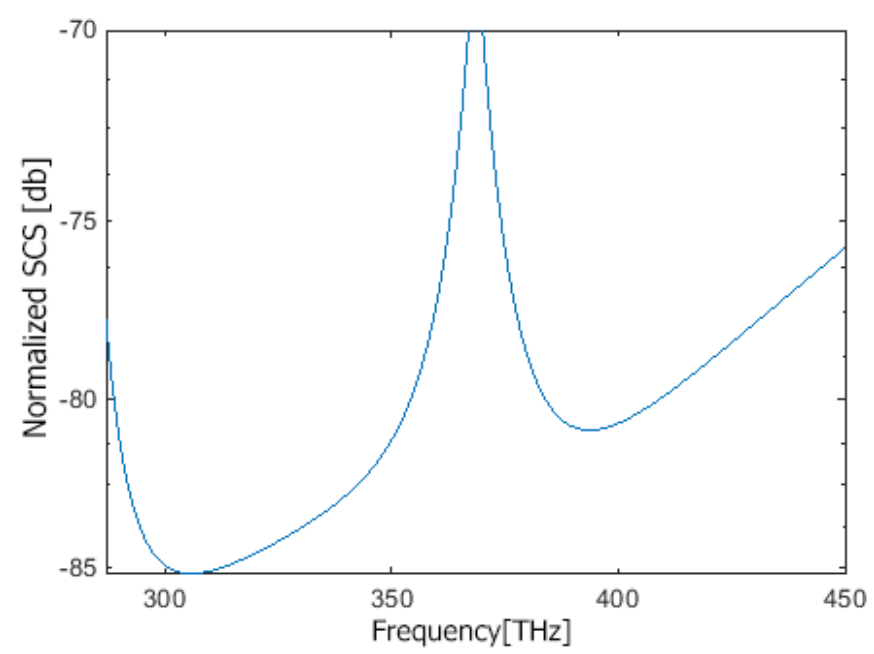

Fig. 4. Normalized SCS of the dielectric cylinder with radius 10nm covered by two plasmonic layers.

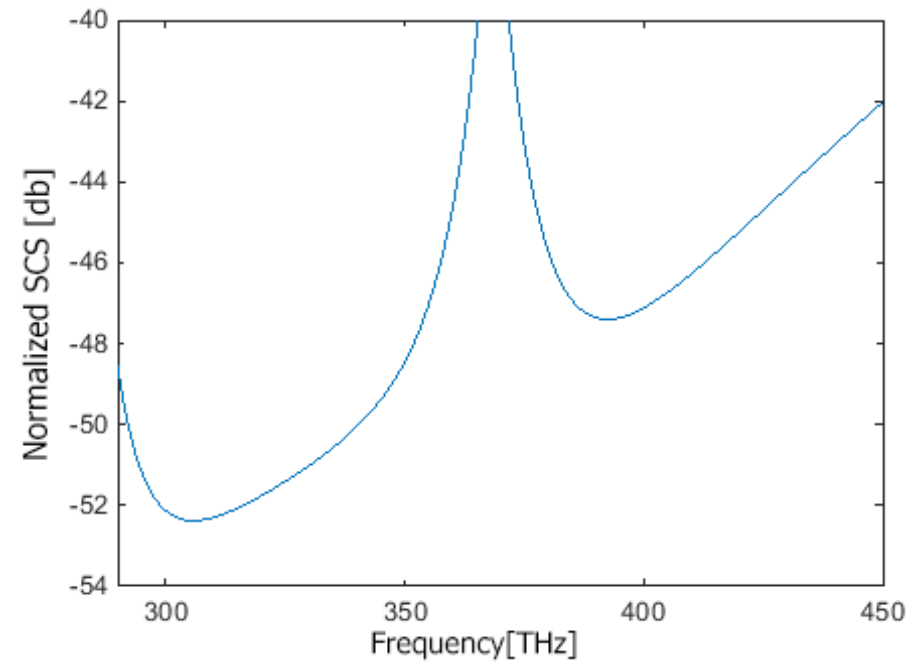

Fig. 5. Normalized SCS of the dielectric cylinder with radius $30 \mathrm{~nm}$ covered by two plasmonic layers.

It can be concluded that by using two cloaking layers, invisibility is obtained at two frequencies. It is also observed that scattering reduction reduces for higher frequencies as a result of increasing $k o a$ and the object becomes electrically larger. Therefore, increasing the higher harmonics of scattering coefficients leads to the reduction of cloaking performance, which is completely matched with the conclusions of reference [11] for spherical cloaks.

To better understand the performance of a plasmonic cloak, it is worth examining the behavior of the normalized SCS of central cylinder with respect to relative electric permittivity of region 2 in Fig. 3 by changing the electric permittivity of it. (Fig. 6) 


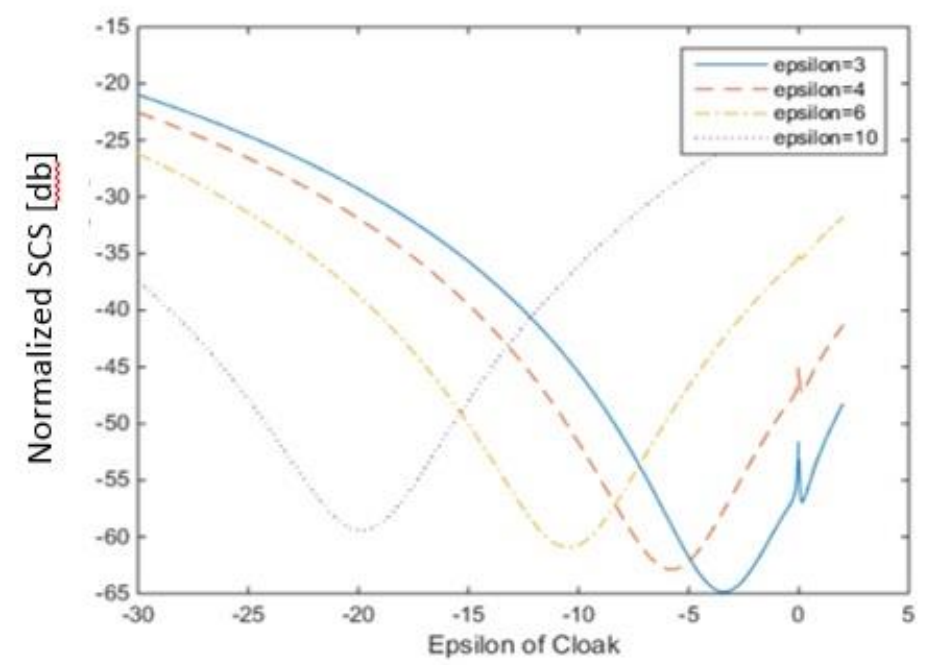

Fig. 6. Normalized SCS of the dielectric cylinder with radius $100 \mathrm{~nm}$ with respect to relative electric permittivity of the first cloak for different electric permittivity of central cylinder.

In this case the radius of central cylinder is chosen $\mathrm{a}=100 \mathrm{~nm}$, the radii of plasmonic cloaking layers $\mathrm{a}_{\mathrm{c} 2}=1.1 \mathrm{a}$ and $\mathrm{a}_{\mathrm{c} 3}=$ 1.2a respectively, $k_{0}$ a $=0.1 \mathrm{rad}$. and relative electric permittivity of cloaked cylinder is chosen $3,4,6$ and 10 .

As can be seen, for a cylinder with a higher electric permittivity, cloaks with a more negative electric permittivity are needed. As a result, the performance of the cloaks becomes weaker, which is quite obvious. The reason for this is that with increasing the electric permittivity of the cylinder, the scattering coefficients become larger and so invisibility becomes more difficult and therefore the performance of plasmonic cloaks also decreases.

At this step, it is appropriate to examine the performance of the cylindrical cloak in relation to the changes in the radius of the central cylinder in Fig. 3. Fig. 7 shows the values of normalized SCS in terms of the relative electric permittivity of region 2 for the radii of its central cylinder a=10, 50,100\&500 nm at frequency $100 \mathrm{THz}$.

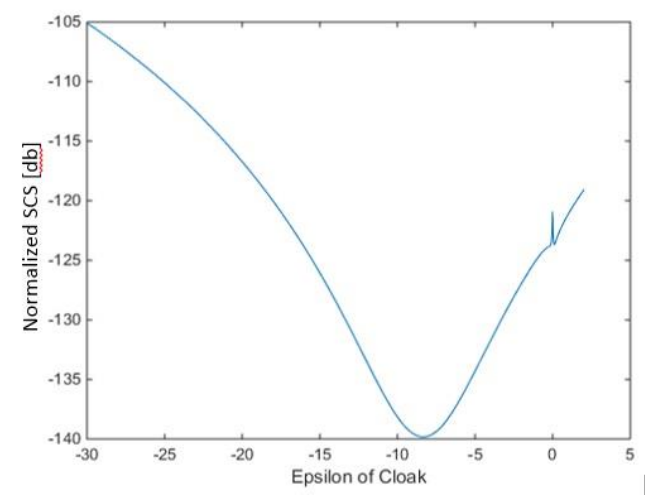

(a)

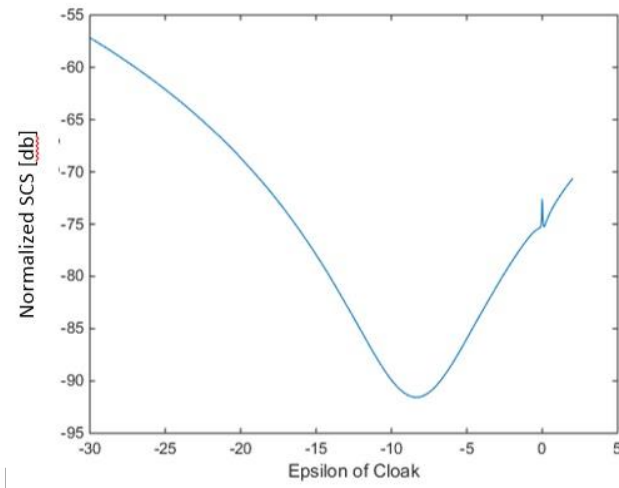

(b) 


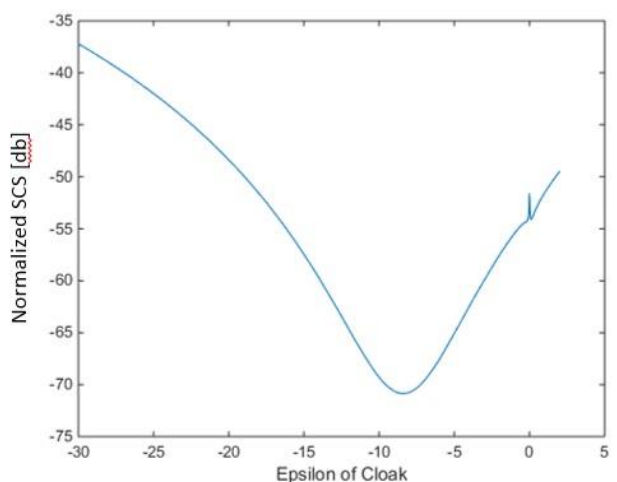

(c)

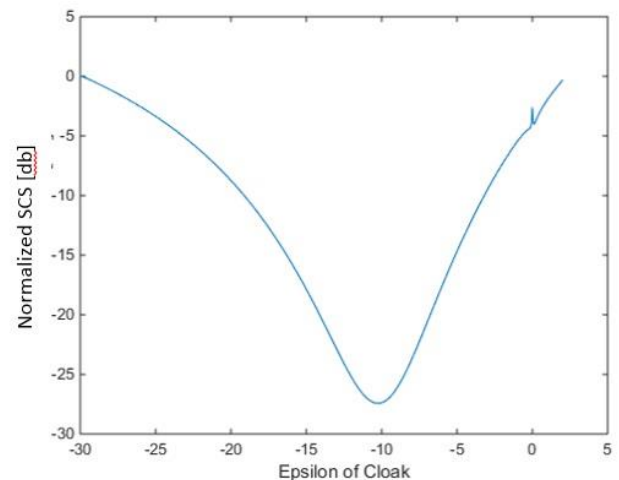

(d)

Fig. 7. Normalized SCS of the dielectric central cylinder with radii (a) $10 \mathrm{~nm}$, (b) $50 \mathrm{~nm}$, (c) $100 \mathrm{~nm}$, (d) $500 \mathrm{~nm}$, with respect to relative electric permittivity of the first cloak.

As can be seen, the normalized SCS decreases sharply for certain values of the relative electric permittivity of cloak, and this occurs for all radii of the central cylinder. These values of the relative electric permittivity of cloak are all negative. So it is possible to minimize the scattering coefficients in Equation (6) by using plasmonic or metamaterial cloaks, and thus achieve transparency. It is also observed that the drop rate of normalized SCS decreases with increasing radius of the central cylinder. This phenomenon is due to the appearance of higher terms in the SCS relation and is consistent with the results stated in reference [18] for the performance of spherical cloaks. Also, the drop location of normalized SCS in Fig. 7(a \& b) is almost the same for cylinders with small radii, and by increasing the radius of the cylinder in Fig. 7(c \& d), the values of the relative electric permittivity of cloak change at the drop locations of SCS. The reason for this is that for cylinders with a smaller radius, the Bessel functions in the scattering coefficient relations (Equation 6), can be approximated by a small argument approximation, and finally a closed form can be obtained to minimize the scattering coefficients. Since this closed form is a function of the ratio of the radius of the cylinder to the radius of the cloak, and here this ratio is the same in all the design of Fig. 7, it is almost the same for small cylinders, but with increasing radius of the cylinder this approximation is not valid. Then the drop location of SCS changes with changing the radius of the cylinder.

The main advantage of the cloaking structure that has been proposed in this paper in comparison to the other papers in the literature, that's it, in previous articles, the study of multilayer cloaking structures has not been done analytically and with precise mathematical equations, and this has been done only for the two-layer structure, while in this article the analysis of these structures has been done completely accurately and using mathematical support. Obviously, in the particular case, this precise mathematical analysis can also be used for two-layer structures.

Table. 1 compares different cloaking techniques. It shows that by considering the radius of the cylinder, RCS reduction and multi-layer and multi-frequency cloak, the presented method in this paper has a good performance. 
Table.1 Comparison of the cloaking methods in the literature with this work.

\begin{tabular}{|c|c|c|c|c|}
\hline References & Radius of the Cylinder & RCS Reduction & Cloaking Method & $\begin{array}{c}\text { Multi-Layer } \\
\text { And } \\
\text { Multi-Frequency }\end{array}$ \\
\hline$[21]$ & $0.1 \lambda$ & $10 \mathrm{~dB}$ & Plasmonic Cloaking & $x$ \\
\hline [22] & $0.13 \lambda$ & $13 \mathrm{~dB}$ & Mantle Cloaking & $x$ \\
\hline [23] & $0.13 \lambda$ & $8 \mathrm{~dB}$ & $\begin{array}{c}\text { Core-Shell } \\
\text { Nanoparticles }\end{array}$ & $x$ \\
\hline [24] & $0.12 \lambda$ & $12 \mathrm{~dB}$ & Graphene Metasurface & $x$ \\
\hline [25] & $0.1 \lambda$ & $13.5 \mathrm{~dB}$ & Graphene Monolayer & $x$ \\
\hline [14] & $0.2 \lambda$ & $10 \mathrm{~dB}$ & Plasmonic Cloaking & $\checkmark$ \\
\hline This Work & $0.17 \lambda$ & $26 \mathrm{~dB}$ & Plasmonic Cloaking & $\checkmark$ \\
\hline
\end{tabular}

\section{Conclusion}

In this paper, it was investigated that the presence of metamaterial or plasmonic cloaks with a negative or near zero relative electric permittivity can reduce the scattering of the structure by creating reverse polarization and thus create invisibility.

The design procedure of multilayer plasmonic cloak for invisibility of a dielectric infinite cylinder has been investigated. Also in this article the analysis of these structures has been done completely accurately and using mathematical support. Simulation results for SCS of cloaked cylinders with two plasmonic layers are also shown which verifies two cloaking operation frequencies. Also behaviour of the normalized SCS of central cylinder with respect to its radius and electric permittivity is investigated.

\section{References}

[1] Dolin, L. S.: To the Possibility of Comparison of Three-Dimensional Electromagnetic Systems With Nonuniform Anisotropic Filling. Izvestiya Vuzov Radiofizika, 4(5), 964-967 (1961).

[2] Pendry, J. B., Schurig, D., Smith, D.R.: Controlling Electromagnetic Fields. Science, 312, 1780-1782 (2006).

[3] Greenleaf, A., Kurylev, Y., Lassas, M., Uhlmann, G.: Cloaking Devices, Electromagnetic Wormholes, and Transformation Optics. SIAM Review, 51(1), 3-33 (2009).

[4] Kerker, M.: Invisible bodies. Journal of Optical Society of America, 65(4), 376-379, (1975).

[5] Alù, A., Bilotti, F., Vegni, L.: Method of Lines Numerical Analysis of Conformal Antennas. IEEE Transactions on Antennas and Propagation, 52(6), 1530- 1540, (2004).

[6] Alitalo, P., Luukkonen, O., Jylha, L., Venermo, J., Tretyakov, S.A.: Transmission-Line Networks Cloaking Objects From Electromagnetic Fields. IEEE Transactions on Antennas and Propagation, 56(2), 416-424 (2008). 
[7] Alitalo, P., Ranvier, S., Vehmas, J., Tretyakov, S.: A Microwave Transmission-Line Network Guiding Electromagnetic Fields through a Dense Array of Metallic Objects. Metamaterials, 2(4), 206-212 (2008).

[8] Alitalo, P., Culhaoglu, A.E., Osipov, A.V., Thurner, S., Kemptner, E., Tretyakov, S.A.: Bistatic Scattering Characterization of a Three-Dimensional Broadband Cloaking Structure. Journal of Applied Physics, 111(3), 034901-5 (2012).

[9] Nicorovici, N.A., Milton, G.W., McPhedran, R.C., Botten, L.C.: Quasistatic Cloaking of Two dimensional Polarizable Discrete Systems by Anomalous Resonance. Optics Express, 15(10), 6314-6323 (2007).

[10] Nicorovici, N.P., McPhedran, R.V., Enoch, S., Tayeb, G.: Finite wavelength cloaking by Plasmonic resonance. New Journal of Physics, 10(11), 115020 (2008).

[11] Alù, A., Engheta, N.: Achieving Transparency with Plasmonic and Metamaterial Coatings. Physical Review E, 72(1), 016623 (2005).

[12] Johnson, P.B., Christy, R.W.: Optical Constants of the Noble Metals. Physical Review B, 6(12), 4370-4379 (1972).

[13] Chew, H., Kerker, M.: Abnormally low electromagnetic scattering cross sections. Journal of Optical Society of America, 66(5), 445 (1976).

[14] Alù, A., Engheta, N.: Theory and Potentials of Multi-Layered Plasmonic Covers for Multi-Frequency Cloaking. New Journal of Physics, 10, 115036 (2008).

[15] Alù, A., Engheta, N.: Plasmonic Materials in Transparency and Cloaking Problems: Mechanism, Robustness, and Physical Insights. Optics Express, 15(6), 3318-3332 (2007).

[16] Alù, A., Engheta, N.: Cloaking and Transparency for Collections of Particles with Metamaterial and Plasmonic Covers. Optics Express, 15(12), 7578-7590 (2007).

[17] Alù, A., Engheta, N.: Cloaking a Sensor. Physical Review Letters, 102, 233901 (2009).

[18] Farhat, M., Rockstuhl, C., Bagci, H.: A 3D Tunable and Multi-Frequency Graphene Plasmonic Cloak. Optics Express, 21(10), 12592-12603 (2013)

[19] Alù, A., Rainwater, D., Kerkhoff, A.: Plasmonic Cloaking of Cylinders: Finite Length, Oblique Illumination and Cross-Polarization Coupling. New Journal of Physics, 12, 103028 (2010).

[20] Balanis, C.A.: Advanced Engineering Electromagnetics. John Wiley and Sons (1989).

[21] Bilotti F, Tricarico S, Vegni L.: Electromagnetic Cloaking Devices for TE and TM Polarizations. New J Phys 12, 115035 (2008).

[22] Danaeifar M, Booket M, Granpayeh N.: Optical Invisibility of Cylindrical Structures and Homogeneity Effect on Scattering Cancellation Method. Electron Lett, 52, 29-31 (2016).

[23] Monti A, Bilotti F, Toscano A.: Optical Cloaking of Cylindrical Objects by Using Covers Made of Core-Shell Nanoparticles. Opt Lett, 36, 4479-81 (2011).

[24] Chen PY et al.: Nanostructured Graphene Metasurface for Tunable Terahertz Cloaking. New J Phys, 15, 123029, (2013).

[25] Hamzavi-Zarghani Z, Yahaghi A, Matekovits L.: Electrically Tunable Mantle Cloaking Utilizing Graphene Metasurface for Oblique Incidence. International Journal of Electronics and Communications (AEÜ), 116, 153080 (2020). 